\title{
Effect of Fat Content on Water Sorption Properties of Biscuits Studied by Nuclear Magnetic Resonance
}

\author{
Fa-yi Hao", Li-xin Lu ${ }^{1,2, *}$, Chang-feng Ge ${ }^{3}$ \\ ${ }^{1}$ Jiangnan University, Wuxi, P.R. China \\ ${ }^{2}$ Jiangsu Key Laboratory of Advanced Food Manufacturing Equipment and Technology, Wuxi, P.R. China \\ ${ }^{3}$ Rochester Institute of Technology, 78 Lomb Memorial Drive, Rochester, NY 14623, USA \\ *Corresponding author: lulx@jiangnan.edu.cn
}

Received September 01, 2014; Revised October 24, 2014; Accepted October 26, 2014

\begin{abstract}
Moisture sorption isotherm is a well established method to characterize water sorption properties and behavior of food materials. However, this approach doesn't adequately reflect the molecular mobility that taking place during water sorption process. Nuclear magnetic resonance (NMR) can provide information about the water mobility and molecular interactions between water and food components. The biscuits with different fat addition were studied using water sorption isotherm and ${ }^{1} \mathrm{H}$ low-field NMR at $25^{\circ} \mathrm{C}$ and water activity ranging from 0.2 to 0.90 , the changes in equilibrium moisture content, transverse relaxation time $\left(\mathrm{T}_{2}\right)$ and proton intensity of biscuits were defined. The $T_{2}$ were measured with Carr-Purcell-Meiboom-Gill (CPMG) sequences. It was demonstrated that fat content of biscuits influenced directly the equilibrium moisture content and water status. One or two water populations were observed as the water activity increased, each of which had a distinct relaxation time $T_{2}$ or molecular mobility. The relaxation time manifested that with the increase of fat addition, the water inside the samples became more mobile, and proton intensity indicated that the amount of water uptake decreased with increasing fat addition. The low-field NMR was demonstrated to provide complementary interpretation to that of water sorption isotherm.
\end{abstract}

\section{Keywords: Nuclear magnetic resonance, water sorption isotherm, transverse relaxation time, biscuit, fat content}

Cite This Article: Fa-yi Hao, Li-xin Lu, and Chang-feng Ge, "Effect of Fat Content on Water Sorption Properties of Biscuits Studied by Nuclear Magnetic Resonance." Journal of Food and Nutrition Research, vol. 2, no. 11 (2014): 814-818. doi: 10.12691/jfnr-2-11-9.

\section{Introduction}

Water is well recognized as a critical component in food as it plays a fundamental role not only in the food processing operations but also in defining quality and stability of foods. The water availability and distribution inside the foods continue to change during storage and therefore are crucial to determine shelf life of foods [1,2].

A common approach in measuring water availability in foods is that of water activity. Moisture sorption isotherm, which describes the relationships of water activity and moisture contents, is a well established method to characterize water sorption properties and behavior of food materials [3]. The nature of this relationship depends on the interaction between water and food composition, in particular, several authors have pointed out the influence of fat addition on the moisture sorption behavior in sponge cake [4,5] and crackers [6].

Furthermore, the mathematical interpretation of water sorption isotherms by Guggenheim-Anderson-de Boer (GAB) model has received much attention due to its relationship with shelf life of foods. The typical moisture sorption isotherm can be divided into three regions depending on the state of water present. In monolayer sorption region, water molecules may be bound to an ionic group such as a carboxyl or an amino group. In multilayer sorption region, adsorption of additional layers over the monolayer occurs and water binding is assumed to consist of water molecules bonded by hydrogen bonds to hydroxyl and amide groups. The third region corresponds to condensation of water in the pores of the food material [7]. However, the water sorption isotherm does not reflect the molecular mobility taking place during water sorption and physical states of water inside the foods. The understanding of food-water relationships and molecular mobility of water inside the foods could complement the information provided by water sorption isotherms [8].

Nuclear magnetic resonance (NMR) is a powerful technique in food science with the ability to provide information about the water mobility and molecular interactions between water and food components [9]. In particular, low-field NMR spectroscopy has been applied to study the molecular mobility in a wide range of complex foods such as cheeses [10,11], bread [12,13], coffee [14] and also in pasta matrixes [15], in an attempt to relate molecular mobility to quality and stability of foods. The combination of NMR relaxation time and water sorption isotherm can thus allow providing a multilevel portrait of the water behavior in foods that govern the stability of food system. 
It was revealed that ${ }^{1} \mathrm{H}$ transverse relaxation time $\mathrm{T}_{2}$ is able to reflect the availability and mobility of water inside the foods [16,17]. Monolayer of water molecules is almost immobilized, and it behaves so in many aspects (also in NMR spectroscopy) as part of a solid. As more water is absorbed by foods, additional layers of water link to monolayer in order to form the multilayer water. $\mathrm{T}_{2}$ can be used to indicate the mobility of these water layers, to identify each layer with different relaxation times (strongly tied up or structured, and weakly tied up according to water bond energy). For example, monolayer water molecules directly linked to macromolecules by hydrogen bonds usually presents a $T_{2}$ of dozens of microseconds, while the multilayer water molecules shows a $\mathrm{T}_{2}$ of dozens to hundreds of milliseconds.

However, few studies have been reported about the mobility of molecules in complex systems containing fat. Reference [18] studied biscuit dough using NMR relaxation and found that there were four water populations can be distinguished. Reference [19] reported several transverse relaxation times $T_{2}$ were obtained from transverse relaxation curves of baked cake, and then attributed to the solid phase, aqueous phase and lipid phase, respectively. The NMR relaxation signal from a complex product such as biscuit is hard to interpret because of its multi-exponential behavior, and attribute the relaxation components to each phase (solid, fat, water ...) is difficult.

The interest of this study was to a) investigate the impact of fat addition on the water sorption characterization of food products; b) further understand interactions between the food components and water using low-field pulsed NMR; c) determine the change of water mobility during sorption process so as to gain insight into water sorption behavior at the molecular level and provide a complementary interpretation for that of water sorption isotherm.

\section{Materials and Methods}

\subsection{Preparation of Biscuit Samples}

Fermented biscuits of varying fat content were prepared in the laboratory. The compositions were as shown in Table 1 .

Table 1. Biscuits Composition

\begin{tabular}{|c|c|c|c|c|}
\hline & & $\mathrm{Z0}$ & $\mathrm{Z8}$ & Z16 \\
\hline \multirow{7}{*}{ Composition (g) } & Flour & 100 & 100 & 100 \\
\hline & Water & 36 & 34 & 32 \\
\hline & Corn oil & 0 & 8 & 16 \\
\hline & Sugar & 3 & 3 & 3 \\
\hline & Dry yeast & 1.5 & 1.5 & 1.5 \\
\hline & Slat & 0.8 & 0.8 & 0.8 \\
\hline & Baking soda & 0.8 & 0.8 & 0.8 \\
\hline
\end{tabular}

The three studied biscuits were called hereafter Z0, Z8 and Z16. The biscuits were prepared as follows: dry yeast (Angelyeast, Wuchang, China) was dissolved firstly with warm water of $40^{\circ} \mathrm{C}$ and stirred for activation. Flour (American Rose Serials, Lam Soon, HongKong, China), salt, sugar, baking soda, water and corn oil (Yihai Kerry, Shanghai, China)were mixed in Murenking (Shenzhen Muren Appliance, China) mixer for 2 min at speed 2, then add the activated yeast with moderate agitation for $7 \mathrm{~min}$ at speed 3. The dough was rested in a plastic film at $34^{\circ} \mathrm{C}$ for $30 \mathrm{~min}$, and then laminated into slaps of $2 \mathrm{~mm}$ thickness and cut with a circular pastry cutter (25 mm of diameter). The sheets were proofed at $34^{\circ} \mathrm{C}, 90 \%$ relative humidity for $40 \mathrm{~min}$. After baking for $12 \mathrm{~min}$ in an oven at $175^{\circ} \mathrm{C}$, the cookies were allowed to cool at ambient temperature for $30 \mathrm{~min}$ and then stored into waterimpermeable plastic pouches.

\subsection{Water sorption experiments}

Water sorption measurements of biscuits were performed at $25^{\circ} \mathrm{C}$ using Aqualab Vapor Sorption Analyzer (Decagon Devices, Pullman, WA, USA). The biscuits were pre-equilibrated in desiccators contained $\mathrm{P}_{2} \mathrm{O}_{5}$ for at least $5 \mathrm{~d}$ [5]. The thoroughly dried biscuits were put into the instrument for moisture sorption experiment. The initial water sorption range was set at water activity of $0.1-0.2$. It was assumed that the equilibrium was reached when the change in sample mass as a function of time was lower than $0.002 \% / \mathrm{min}$. And the next sorption range was $0.2-0.3$ water activity, and so on, the last sorption range was $0.8-0.9$. The weight of samples at each water activity were recorded, and the equilibrium moisture content was calculated for water sorption isotherm.

\subsection{Modeling of sorption isotherm}

The mathematical form of the GAB model is:

$$
M_{e}=\frac{m_{0} C K a_{w}}{\left(1-K a_{w}\right)\left(1-K a_{w}+C K a_{w}\right)}
$$

Where $\mathrm{m}_{0}$ is the monolayer value, $C$ and $K$ are the kinetic constants related to the sorption, $M_{e}$ is the equilibrium moisture content at each $\mathrm{a}_{\mathrm{w}}$.

The model for sorption isotherm was simulated using Matlab software (The Mathworks, Inc., Natick, MA, USA) by minimizing the root mean square deviations between simulated and experimental results using the LevenbergMarquardt procedure. The goodness of fit was evaluated with the coefficient of determination(R-squared) and root mean square error (RMSE) between the experimental and predicted moisture content:

$$
\begin{aligned}
R-\text { squared } & =1-\frac{\sum_{i=1}^{N}\left(m_{e}-m_{p}\right)^{2}}{\sum_{i=1}^{N}\left(m_{e}-\bar{m}\right)^{2}} \\
R M S E & =\sqrt{\frac{\sum_{i=1}^{N}\left(m_{e}-m_{p}\right)^{2}}{N}}
\end{aligned}
$$

Where $m_{e}$ is the experimental value, $m_{p}$ is the predicted value, $\bar{m}$ is the mean of experimental data and $\mathrm{N}$ is the number of experimental data.

\subsection{NMR experiments}

NMR experiments were measured by PQ001 low-field NMR Analyzer (Shanghai Niumag Instruments, China) with a $0.55 \mathrm{~T}$ magnetic field operating at a resonance 
frequency of 21.3 MHz. Biscuits that reached equilibrium at each water activity range were immediately for NMR experiments after weighing. The biscuit samples were placed into a glass tube (25 $\mathrm{mm}$ in diameter) and sealed the open end to prevent moisture loss.

Transversal relaxation times $\mathrm{T}_{2}$ of was measured by Carr-Purcell-Meiboom-Gill (CPMG) pulse sequence, comprising a $90^{\circ}$ pulse in a specific radio frequency, followed by a series of pulses in $180^{\circ}$ phase. The parameters of CPMG sequence were as follows: $90^{\circ}$ pulse time $=4.1 \mu \mathrm{s}, 180^{\circ}$ pulse $=8.8 \mu \mathrm{s}$, repeated waiting time $=2500 \mathrm{~ms}$, echo time $=0.2 \mathrm{~ms}$, echo count $=6000$, number of sampling=32. Transverse relaxation data were analyzed with the following models [13]:

$$
I_{C P M G}(t)=\sum_{i=1}^{n} A_{i} \exp \left(-t / T_{2 i}\right)
$$

Where $T_{2 i}$ and $A_{i}$ are the relaxation time and the proton intensity that corresponding to $\mathrm{T}_{2 \mathrm{i}}$ state. Transverse relaxation curves obtained from CPMG pulse sequences using an iterative optimization method were fitted with analysis software from Niumag Instruments.

\section{Results and Discussions}

\subsection{Water Sorption Isotherm Study}

The moisture sorption experiments of the different biscuits were determined at $25^{\circ} \mathrm{C}$ for water activity ranging from 0.2 to 0.90 . The GAB model was employed to fit the experiment data of Z0, Z8 and Z16 (Figure 1). All the water sorption curves show a sigmoid shape, which is typical of biological products and food materials. GAB equation is extensively employed to describe water sorption in food materials, because of the wide range of water activity of this model applicability [20]. In Table 2 the fitting parameters of the $G A B$ equation are summarized for all the studied biscuits.

Monolayer value or $m_{0}$ which corresponds to the first sorption stage described by GAB equation is slightly higher for $\mathrm{Z} 0$ than for the other two biscuits. The differences in $\mathrm{m}_{0}$ values between biscuits could be due to their different fat proportions (Table 2). GAB monolayer values obtained from water sorption studies are $4.428 \%$, $4.393 \%$ and $4.210 \%$ respectively, for Z0, Z8 and Z16.

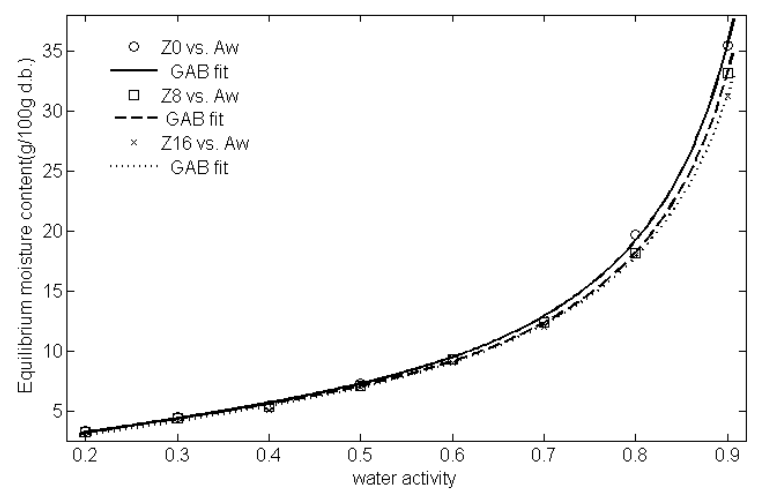

Figure 1. Water sorption isotherms of three biscuits samples at $25^{\circ} \mathrm{C}$

Equilibrium moisture content (EWC) manifested a significant decrease when fat content increased: EWC decreased $6.2 \%$ and $11.8 \%$ for biscuits of Z8 and Z16 compared with $\mathrm{Z} 0$ at 0.9 water activity, respectively. Similar results were observed by other researcher [6] for dry biscuits. When globules of fat surround proteins and starch granules of the food material, it means that an increase of hydrophobic constituents like fat, which binds very little water, would decrease EMC values [21].

Table 2. Fitting Parameters of GAB Models of Three Biscuits With Different Fat Content at $25^{\circ} \mathrm{C}$

\begin{tabular}{cccccc}
\hline \multirow{2}{*}{ Biscuit } & \multicolumn{2}{c}{ GAB parameters } & \multicolumn{2}{c}{ Evaluation index } \\
\cline { 2 - 6 } & $\mathrm{m} 0(\mathrm{~g} / 100 \mathrm{~g})$ & $\mathrm{C}$ & $\mathrm{K}$ & R-squared & RMSE \\
\hline Z0 & 4.428 & 5.661 & 0.9341 & 0.9987 & 0.3361 \\
Z8 & 4.393 & 6.872 & 0.9337 & 0.9982 & 0.1197 \\
Z16 & 4.210 & 5.025 & 0.9428 & 0.9967 & 0.2510 \\
\hline
\end{tabular}

\subsection{Characterization of Water Mobility inside the Biscuits}

Water molecules in food materials can be classified into several fractions according to their molecular mobility or the extent of their association with or "binding" to macromolecules within the material [18]. When water is bound tightly to macromolecules (e.g., protein matrix), it is highly immobilized and shows reduced $\mathrm{T}_{2}$, whereas free water is readily mobile and has relatively long $T_{2}$. The transverse relaxation time $T_{2}$ reflects the mobility of water molecules, so the changes of water state during the water sorption process can be illustrated by $\mathrm{T}_{2}$. More complex systems like foods may include several populations of protons and characterize by multi-exponential relaxation time [11].

Figure 2 shows the distribution of transverse relaxation time $\mathrm{T}_{2}$ obtained by CPMG sequence for biscuits from water activity of 0.2 up to 0.9 . At the lower water activity of 0.2 , there is only one water population was observed for the $\mathrm{Z} 0$ sample with a relaxation time $\mathrm{T}_{2}$ of about $0.123 \mathrm{~ms}$. However, samples with water activity up to 0.7 exhibited another group due the formation of another water population that represents the 'bound water'. The monolayer water molecule formed on macromolecule surface presents very low mobility. And as the water activity increases step by step, the relaxation time becomes longer, which indicates increased mobility of water proton during water sorption process, which meant the gradual formation of multi-layer water molecule. It should be pointed out that for water activity up to 0.8 , an anomaly in the fat curve shape can be noted. Molecular mobility of this anomaly corresponds to the bulk water. At water activity of 0.9 , the transverse relaxation time of biscuit samples manifested three distinct populations, the $\mathrm{T}_{21}$ population with a relaxation time of $0.1-1 \mathrm{~ms}$ can be ascribed to bound water protons, the $T_{22}$ population with a relaxation time of $1-10 \mathrm{~ms}$ can be ascribed to moderate mobile protons, and the main population $\mathrm{T}_{23}$ with a relaxation time of $30-150$ ms can be ascribed to mobile protons in the biscuit samples.

At the end of the spectrum there is an obvious proton group, which represents the signal of the samples' fat contents. The presence of this group was confirmed through the experimental data of $\mathrm{T}_{2}$ obtained in experiments with pure corn oil (Figure 3). Since the biscuits Z8 and Z16 contain some amount of fat, water activity variation has no significant effect on this proton population; therefore, we can assume that the majority of population $\mathrm{T}_{23}$ may attribute to the protons of fat. 

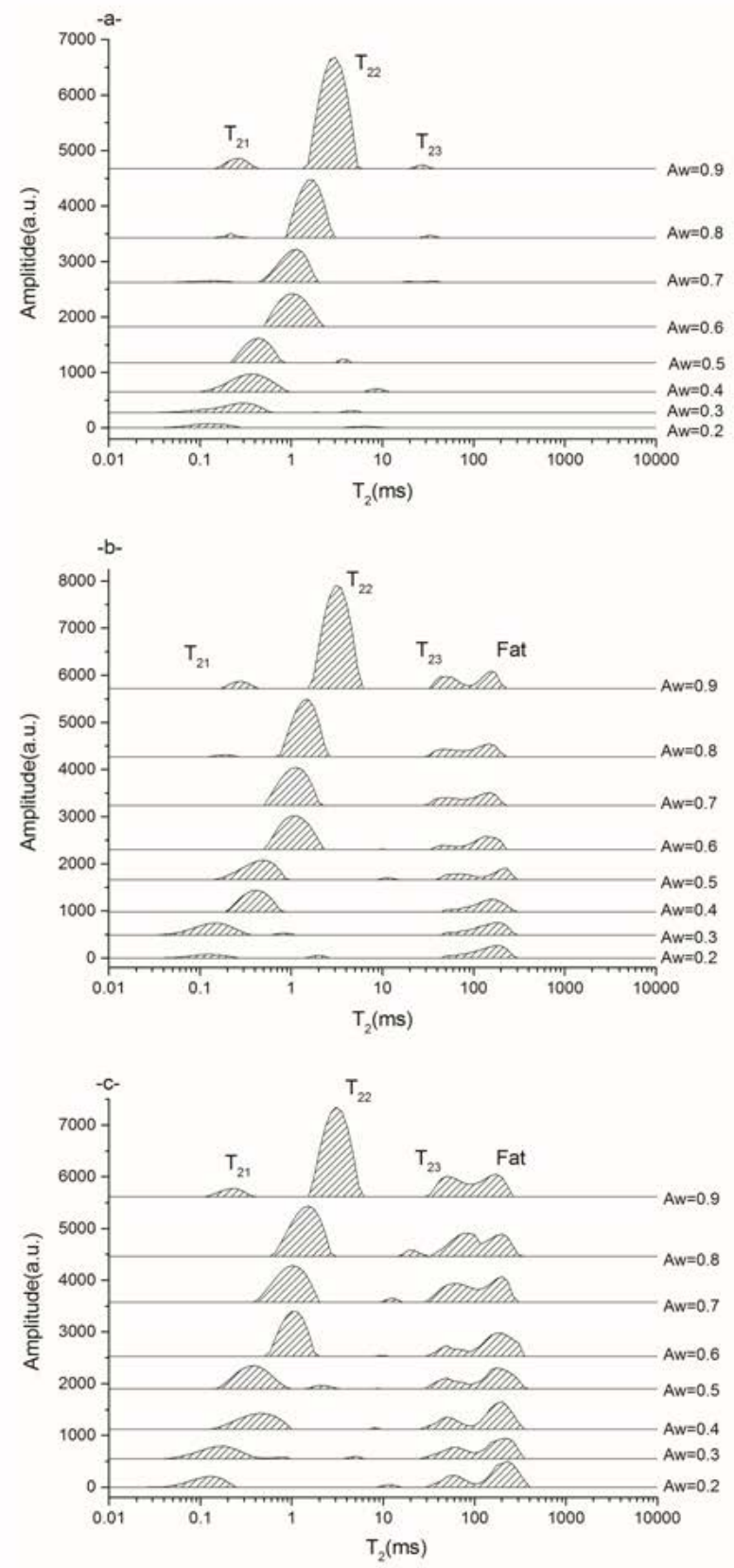

Figure 2. Distribution of $T_{2}$ obtained by CPMG sequence at different water activity (a) Z0 biscuit; (b) Z8 biscuit; (c) Z16 biscuit.

\subsection{Effect of Fat Content on Transverse Relaxation Time $\mathbf{T}_{2}$}

Changes in water mobility are mainly caused by changes in the hydrogen bonding structure. Hydrophilic materials such as proteins and carbohydrates have hydrogen-bonding sites, which are available to form hydrogen bonds with water molecules and thereby influence the water mobility. Higher contents of proteins and/or carbohydrates will decrease the mobility, while lower contents will increase the mobility [22].

Table 3 shows the variations of $\mathrm{T}_{2}$ as a function of water activity, which reflects the mobility of protons increases with increasing water activity. It should be noted that only one water proton population exists inside biscuit samples till water activity of 0.8 besides the fat proton $\mathrm{T}_{23}$. The transverse relaxation time $\mathrm{T}_{2}$ in biscuit $\mathrm{Z} 0$ is faster that that of $\mathrm{Z} 8$ and Z16, which means either the water bonding with other molecules by hydrogen bonding was stronger, or there was more hydrogen-bonding sites in biscuits without fat than that of Z8 and Z16. The increase in fat gave a slower relaxation or more mobile water, indicating that water is bound more strongly to the proteins than that of fat [23].

Table 3. Transeverse Relaxation Time $\mathbf{T}_{2}$ of Biscuit Samples at Different Water Activity

\begin{tabular}{ccccccc}
\hline Water & \multicolumn{2}{c}{ Z0 } & \multicolumn{2}{c}{ Z8 } & \multicolumn{2}{c}{ Z16 } \\
activit & T21(m & T22(m & T21(m & T22(m & T21(m & T22(m \\
y & s) & s) & s) & s) & s) & s) \\
\hline 0.2 & 0.123 & -- & 0.123 & -- & 0.123 & -- \\
0.3 & 0.142 & -- & 0.142 & -- & 0.163 & -- \\
0.4 & 0.376 & -- & 0.433 & -- & 0.433 & -- \\
0.5 & 0.433 & -- & 0.498 & -- & 0.657 & -- \\
0.6 & 0.869 & -- & 1.000 & -- & 1.149 & -- \\
0.7 & 1.000 & -- & 1.000 & -- & 1.322 & - \\
0.8 & 0.187 & 1.322 & 0.215 & 1.519 & 0.327 & 1.519 \\
0.9 & 0.247 & 3.053 & 0.284 & 3.511 & 0.327 & 4.037 \\
\hline
\end{tabular}

\subsection{Intensity of Protons of Biscuits with Different Fat Content}

The intensity of protons from water of different $T_{2 s}$, which are a relative measure of the amount of water corresponding to a certain $\mathrm{T}_{2}$, was also obtained from the NMR experiments. Figure 3 shows that the intensity of proton signal of water in the $T_{2}$ state (including $T_{21}$ and $\mathrm{T}_{22}$ ) increased exponentially over water sorption process. The intensity of proton signal presented a sharp increase trend at 0.7 water activity, according to the hygroscopic nature of biscuit material, which corresponds to the water sorption isotherm. The intensity of proton signal of biscuit Z0 presents the fastest increase, followed by Z8 and Z16, which may attribute to the fat-free nature of $\mathrm{Z0}$, and there exists more water sorption sites, whereas Z8 and Z16 contain more fat, the water uptake of the fat material is very low according to its hydrophobic nature, the hydrophilic groups could tend to group together, and make it less accessible for water molecules [5].

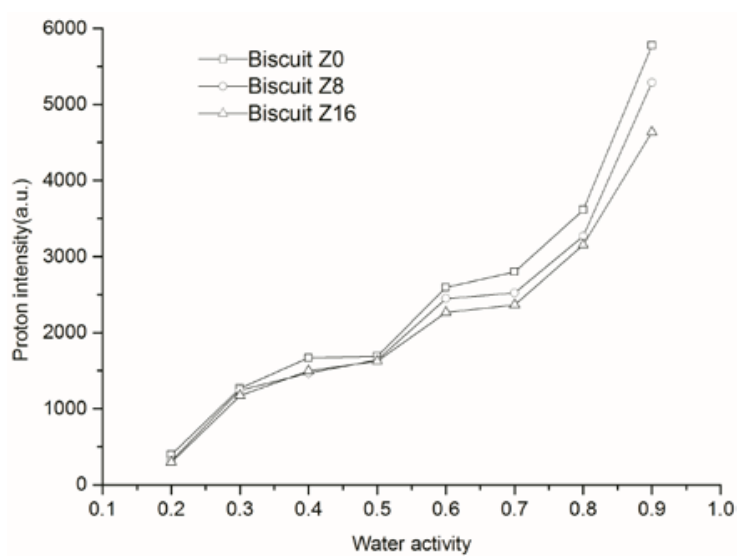

Figure 3. Changes of water proton signal intensity in $T_{2}$ state during water sorption

\section{Conclusions}

The study of water mobility in biscuits with different fat content is a quite challenging subject as the complex composition can affect the distribution of water. However, ${ }^{1} \mathrm{H}$ NMR transversal relaxation times, obtained by CPMG sequences, can be applied for an appropriate definition of 
the different water mobility populations and the effect of fat addition. The moisture sorption isotherm revealed that the equilibrium moisture content can be significantly decreased with addition of fat. One or two water populations were observed using NMR during water sorption process. Changes in $\mathrm{T}_{2 \mathrm{~s}}$ and the corresponding protons intensity suggested that the fat addition influenced the water status and mobility, the biscuits contained fat presented higher water mobility and more dynamic migration of water, and meanwhile, the fat-free biscuit possessed higher protons intensity at each water activity.

\section{Acknowledgements}

This work was supported by the Achievements Industrialization Prospective Research Project of Jiangsu Province grant number BY2013025-26, and by the Fundamental Research Funds for the Central Universities JUSRP51403A.

\section{Competing Interests Statement}

The authors declare that they have no competing financial interests.

\section{References}

[1] Iglesias, H. A. and Chirife, J. Handbook of food isotherms: Water sorption parameters for food and food components. Academic Press Inc, New York, 1982.

[2] Schmidt, S.J. Water and solid mobility in foods. In: Advance in Food and Nutrition Research (Edited by Taylor, S. Nebraska, USA: Academic Press, 2004, 1-101.

[3] Pitombo, R.N.M., Lima, G.A.M.R.. Nuclear magnetic resonance and water activity in measuring the water mobility in Pintado (Pseudoplatystoma corruscans) fish, Journal of Food Engineering, 58, 59-66, 2003.

[4] Roca, E., Guillard, V., Guilbert, S. and Gontard, N. Moisture migration in a cereal composite food at high water activity: effects of initial porosity and fat content. Journal of Cereal Science, 43, 144-151, 2006.

[5] Roca, E., Broyart, B., Guillard, V., Guilbert, S. and Gontard,N. Controlling moisture transport in a cereal porous product by modification of structural or formulation parameters. Food Research International, 40, 461-469, 2007.

[6] Kim, S. S., Kim, S. Y., Kim, D. W., Shin, S. G. and Chang, K. S. Moisture sorption characteristics of composite foods filled with chocolate. Journal of Food Science, 64, 300-302, 1999.

[7] Agudelo-Laverde, L.M., Carolina S.and Maria del P.B.. Proton mobility for the description of dynamic aspects of freeze-dried fruits. Journal of Food Engineering, 125, 44-50, 2014.
[8] Lina, M. A., Carolina, S., and Maria del P.B.. Proton mobility for the description of dynamic aspects of freeze-dried fruits. Journal of Food Engineering, 125, 44-50, 2014.

[9] Hills, B. P. and Nott, K. P. NMR study of water compartmentation in carrot parenchyma tissue during drying and freezing. Applied Magnetic Resonance, 17, 521-535, 1999.

[10] Aylin, A., Mecit, H. O., Kathryn, L. M. and Michael J. M. Monitoring changes in feta cheese during brining by magnetic resonance imaging and NMR relaxometry. Journal of Food Engineering, 107, 200-207, 2011.

[11] Budiman, M., Stroshine, R.L., and Cornillon, P. Moisture measurement in cheese analogue using stretched and multiexponential models of the magnetic resonance $\mathrm{T}_{2}$ relaxation curve. Journal of Dairy Research, 69, 619-632, 2002.

[12] Alessia, L., Amir, M.A. and Yael, V. Characterization of water distribution in bread during storage using magnetic resonance imaging. Magnetic Resonance Imaging, 25, 1449-1458, 2007.

[13] Chen, P. L., Long, Z., Ruan, R. and Labuza, T. P. Nuclear magnetic resonance studies of water mobility in bread during storage. Lebensmittel-Wissenschaft and Technology, 30, 178-183, 1997.

[14] Mateusa, M.L., Championb, D., Liardona, R. and Voilley, A. Characterization of water mobility in dry and wetted roasted coffee using low-field proton nuclear magnetic resonance, Journal of Food Engineering, 81, 572-579, 2007.

[15] Carini, E., Vittadini, E., Curti, E., Antoniazzi, F.\&Viazzani, P. Effect of different mixers on physicochemical properties and water status of extruded and laminated fresh pasta. Food Chemistry, 122, 462-469, 2010.

[16] Butz, P., Hofmann, C. and Tauscher, B. Recent developments in noninvasive techniques for fresh fruit and vegetable internal quality analysis. Journal of Food Science, 70, R131-R141, 2005.

[17] Ruan, R. R., Wang, X., Chen, P. L., Fulcher, R. G., Pesheck, P.\& Chakrabati, S. Study of water in dough using nuclear magnetic resonance. Cereal Chemistry, 76, 231-235, 1999.

[18] Assifaoui,A., Champion, D., Chiotelli, E., and Verel, A. "Characterization of water mobility in biscuit dough using a lowfield 1H NMR technique.” Carbohydrate Polymers, 64,197-204, 2006.

[19] Le Grand, F., Cambert, M., Mariette, F. NMR Signal Analysis To Characterize Solid, Aqueous, and Lipid Phases in Baked Cakes, Journal of Agricultral and Food Chemistry, 55, 10947-10952, 2007.

[20] Furmaniak, S., Terzyk, A.P., Goembiewski, R., Gauden, P.A., Czepirski,L. Searching the most optimal model of water sorption on foodstuffs in the whole range of relative humidity, Food Research International, 42, 1203-1214, 2009.

[21] Baltsavias, A., Jurgens, A., van Vliet. T. Fracture Properties of Short-Dough Biscuits: Effect of Composition, Journal of Cereal Science, 29 (3), 235-244, 1999.

[22] Ruan, R. R., \& Chen, P. L. Water in Foods and Biological Materials: A Nuclear Magnetic Resonance Approach. 1st edition ed. Technomic Publishing Company, Inc, Lancaster, Pennsylvania, 1998.

[23] Andersen, C. M., Frøst, M. B. and Viereck,N. Spectroscopic characterization of low-and non-fat cream cheeses. International Dairy Journal, 20, 32-39, 2010. 